\title{
OBSERVATIONS SUR LE LEXIQUE ISTRO-ROUMAIN ACTUEL
}

Le contact direct entre les Istro-Roumains et les Croates, les nécessités d'ordre social et administratif des Istro-Roumains d'établir un contact permanent aves les institutions officielles, tout comme les différentes situations dans lesquelles ceux-ci se trouvent hors du village ont déterminé et déterminent encore une influence massive de la langue croate (notamment du dialecte ceacavian dans sa variante littéraire) sur l'istro-roumain actuel.

L'analyse d'un certain nombre de textes istro-roumains recueillis en 1982 à Žejane et Sušnjevica nous a permis de constater que la pression exercée par le croate sur le système linguistique istro-roumain vise tous les compartiments de ce dernier, y compris la morpho-syntaxe. ${ }^{1}$ Cette influence se manifeste pourtant d'une manière différenciée en fonction de plusieurs facteurs linguistiques et extralinguistiques, inclusivement psychologiques et sociolinguistiques; elle se reflète non seulement dans de nombreux emprunts, mais aussi dans les innovations istro-roumaines d'après le modèle slave (croate ou slovène), innovations qui, en fin de compte, restent elles aussi des acquisitions de cet idiome en tant qu'éléments alloglotes adaptés au système linguistique istro-roumain.

Sans pouvoir parler de dislocations massives et de la désintégration de compartiments tout entiers du système, on peut observer cependant que le locuteur istro-roumain, bilingue par excellence, passe parfois, sans s'en apercevoir-et surtout dans le plan discursif de la parole-d'un code à l'autre, utilisant en alternance des structures linguistiques hétéroglottes. D'autre part, on peut remarquer, surtout chez les jeunes gens, une tendance accentuée à modifier la base articulatoire sous l'influence du dialecte croate ceacavian, tendence ayant d'importantes répercussions sur la réception correcte des messages par l'enquêteur, même si celui-ci connaît le roumain, le serbo-croate et, partiellement, l'istro-roumain. L'articulation "particulière" dans la prononciation rapide de certains sons se trouvant à la jonction de deux unités lexicales distinctes rend certains énoncés plus difficiles à comprendre.

1 Une typologie des structures morpho-syntaxiques de l'idiome istro-roumain de Žejane, mieux conservées dans le temps, se trouve dans notre ouvrage Observations sur le dialecte istro-roumain de Jeiăn, dans "Studii de dialectologie", Timişoara, 1984. Cf., aussi, Present-Day Tendencies in the Morpho-Syntax of Istro-Romanian Dialect, "Linguistica" XXXI, Ljubljana, 1991, p. 141-154. 
A cause de cela, des mutations apparaissent également sur le plan de la phonétique syntaxique, avec des conséquences prévisibles pour l'évolution de l'idiome, et qui devraient faire l'objet des recherches de spécialistes ayant une formation complexe.

Parmi les éléments de morpho-syntaxe contribuant à la persistance de l'idiome, à sa conservation en tant qu' "île linguistique" dans un milieu fortement influencé par le croate, on peut remarquer tout d'abord les formes bien conservées du paradigme du nom, du pronom et du verbe, dont nous avons parlé en détail dans un autre ouvrage. ${ }^{2}$ Le lexique est cependant soumis aux renouvellements, visibles également dans la morpho-syntaxe, vu qu'en dernière instance c'est toujours par des emprunts que l'on explique les structures morphosyntaxiques de type mixte. En faisant cette affirmation nous pensons à deux situations distinctes qui se manifestent sur le plan des connexions syntagmatiques et qui doivent être strictement délimitées en vue d'une juste appréciation de l'étape d'évolution de l'istro-roumain actuel. Nous avons affaire, en tout premier lieu, à des énoncés où l'on rencontre des éléments croates (slovènes) plus ou moins nombreux, en tant que faits intrinsèques du système linguistique, pénétrés récemment ou à une époque plus lointaine, il s'agit ensuite d'interférences de codes linguistiques conditionnées par les différentes circonstances.

L'analyse des textes figurant dans un recueil paru en 1986 à l'Université de Timişoara nous a permis de conclure que, pour une unité de texte d'environ 3000 mots, les termes d'origine croate ou slovène représentent le tiers des lexèmes utilisés, tandis que les termes d'origine latine constituent les deux autres tiers; des 325 phrases du texte, les syntagmes constitués par la combinaison des eléments d'origine latine et des éléments d'origine slave représentent $38 \%$ par rapport aux syntagmes comprenant seulement des termes slaves $-9,5 \%$; le reste de $25 \%$ est représenté par des constructions syntaxiques dans lesquelles les éléments lexicaux roumains anciens sont structurés d'après le modèle morpho-syntaxique slave. Quant à la fréquence des termes provenant du slave et à leur dispersion dans une unité de texte hétérogène du point de vue thématique, on peut constater une situation bien différente, par rapport au nombre des apparitions lexicales dans la même unité de texte: la fréquence et la dispersion des mots de provenance croate ou slovène sont de beaucoup plus réduites que la fréquence et la dispersion des termes d'origine latine. Par exemple, dans un texte (où l'on parle de sangliers) comprenant 390 mots, 61 unités lexicales sont de provenance croate ou slovène; ces unités se répètent cependant peu de fois dans le texte (şcóda-4 fois, divinu-3 fois, sakí et néca-2 fois, le reste des termes une seule fois). En échange, les mots appartenant au fonds roumain ancien ont une fréquence élévée: des mots comme oånc̆, čésta, ie, porc, ştiĭ, noị, aşå, učide, şi cå, nu de, voị, acmó se répètent quatre fois au moins dans le même texte.

Ainsi, bien que le nombre des termes croates dans le lexique istro-roumain utilisé dans le discours spontané soit abondant - fait établi statistiquement par des spécialistes 
tels que I. Coteanu, ${ }^{3}$ A. Kovačec, ${ }^{4}$ R. Flora ${ }^{5}$ les éléments d'origine latine ont pourtant une fréquence incomparablement plus grande. En ce sens, nous partageons l'opinion formulée par A. Kovačec à partir de son glossaire comprenant 85000 mots istro-roumains, c'est-à-dire que les termes d'origine latine font partie du fonds lexical principal tandis que les mots slaves (croates) appartiennent à la masse du vocabulaire. "Tandis que les verbes slaves sont trois fois plus nombreux que les verbes d'origine romane (166/66), dans le texte on a affaire à la situation inverse: les verbes slaves apparaissent une, deux ou trois fois tout au plus, il arrive rarement qu'ils se répètent plusieurs fois" ${ }^{\prime 6}$, les verbes roumains anciens sont beaucoup plus fréquents dans le texte; les verbes le plus souvent employés, ayant des sens assez imprécis, apportent une information lexicale réduite $(a v e, f i)^{7}$.

Dans ce qui suit, nous allons illustrer - à l'aide des exemples tirés du parler spontané des Istro-Roumains - la fonctionnalité syntagmatique des éléments lexicaux roumains anciens par rapport aux éléments de provenance croate (ou slovène); en même temps nous allons essayer de délimiter les situations contextuelles typiques dans lesquelles fonctionnent les éléments d'origine slave, présents dans différentes combinaisons lexicales "mixtes" bi-, tri-, ou plurimembres.

Le matériel enregistré reflète le fait que la place la plus importante dans les textes istro-roumains actuels sur des thèmes quotidiens revient au lexique roumain ancien; on trouve même des répliques intégralement constituées à partir de ce lexique, ce que l'on peut observer également dans les fragments suivants:

"Pičórele acmó me dóru, că săm betắr. Moånt'e-åm tot lucroåt. Acmó nu pot lucrå că săm bólăn... Cu italiéni åm ân óste fost. Mes pac ân Dunåi fost. An Dunåi am soldat. Åm, åm. Şi tóta Hârvátsca am fost. An Dunåi a lui Frant Íńzef. Ia. Åm, åm." (Turkovit" Miho, 83 ans, Jeiăn).

"Č-åsta-i čå a lu melvę, fråte fil'u. Čå čésta-i fíl'u a lui. Čésta-i unúcu lu a lui. C̆åsta-i č čåsta, spomenícu a lu melvę fil' ča-å-n Austrália murit. Cuin? Lu Vínco? Ačåsta-i Éda. Bíserca lu Dråge când s-a meritåt. Ča ie čåsta? Čåsta smo toţ. Čåsta åm io io slikęit. Č̆åsta-i fil'a lu fråtele" (Sankovit" Katarina, 81 ans, Jeiăn).

3 Cf. Cum dispare o limbă (istroromâna), Bucureşti, 1957, p. 20-25. L'auteur remarque le fait que, du point de vue de leur origine, plus de $30-35 \%$ des termes appartenant au fonds principal lexical istro-roumain sont de provenance croate ou slovène.

4 Cf. Descrierea istroromânei actuale, Bucureşti, 1971, p. 202-203.

5 D'après l'interprétation statistique d'un texte réduit comme dimensions, l'auteur établit une proportion de $50 \%$ d'éléments slaves et $50 \%$ d'éléments latins.

6 Idem, ibidem.

7 Fait remarqué par André Martinet: "Plus les unités sont fréquentes dans un système, moins elles apportent d'information et vice-versa: lorsque la fréquence d'une unité diminue, son contenu informationnel augmente automatiquement. (Éléments de linguistique générale, Paris, Armand Colin, 1967, p. 194). 
"Lúcru â ân Opatîñe. Ča lúcru oånča? E, şå, tot: ân gostílne, ujés. Cu cârcoåta, ân cârcă cu brénta åm åpa purtåt... Şi-n sélişte meg. Şi våc-am avút. Vândút-am våca. Acmó am sámo doi câr şi måčca" (Sancovit" Mila, 47 ans, Jeiăn).

"Verít-am co̊åsa şi ópet am počnít lucrå ân covaţie. Cu carbúru şi cu lę́mnele şi cu iein napravljåem, cu ómiri, cu secúre, cosíru, sapúne, vózu, ali våčile. Şi lucråm şi ân pemíntu, lucråt-am. Ali oåstaz io sem ân pezión, a tóturo maréçis pamíntu lucrå ke ţíre ân pemínt lúcră şi vęra, ie ân vęra (...), ie âi sâr". (Fráne Belúlović, 61 ans, Suşnieviţa).

"Ţe tu plânji muşåta féta/Dup-ásta muşåta zi?

Ion plâng şi ấnca voi plấnje/Fråieru nu vrêxa se verí" (F. Belúlović).

En ce qui concerne la présence de l'élément croate (ou slovène) dans des monologues ou bien dans des répliques de différents dialogues, une constatation s'impose dès le début: tous les éléments slaves n'ont pas la même importance fonctionnelle dans le plan discursif du parler des Istro-Roumains d'aujourd'hui; de même, toutes les acquisitions - qu'elles soient anciennes ou plus récentes - ne laissent pas en égale mesure leur empreinte sur la structure de l'idiome, pour déterminer son évolution ultérieure. Tandis que certains emprunts se sont fixés dans le parler des Istro-Roumains, ayant une fonctionnalité élevéé, d'autres emprunts ne peuvent être considérés que des interférences temporaires de codes linguistiques, justifiées par le contexte situationnel plus large.

C'est pourquoi il nous faut préciser quelles sont les situations les plus importantes où il y a concurrence entre les structures lexicales de type slave et celles de type roumain.

a) On distingue, d'une part, dans le parler des Istro-Roumains, les situations où des éléments d'origine croate ou slovène appartenant à la langue officielle sont empruntés tels quels, sans aucune adaptation au système istro-roumain: úpala plút'a "congestion pulmonaire", zráčna liniża "trajet aérien", ósnovna şcóla "école élémentaire", vişe l'údi "plusieurs hommes", iáko bólna "très malade", lóvačco drújstvo "association des chasseurs", vélike vlásti "les grandes puissances", filozófski facultét "faculté de philosophie" etc.

Une situation similaire peut être constatéé dans le dialecte de la population allemande du Banat: "Tes is vaişamer" (d'après une information fournie par Peter Kottler).

b) Une place distincte dans le discours élaboré est occupée par les acquisitions anciennes ou plus récentes de l'idiome: lo pol de beséde poịedés "Moi j'avale les mots à demi"; Ma íno săm colę zadujita "J'y ai des obligations"; stopedéset métri "Cent cinquante mètres"; måia şi čåia "mère et père"; Noi podugúím réčile "Nous traînons les mots"; Acmo's volítvele "Maintenant il y a les élections" et bien d'autres. Cf. Ich han nă lămurirt dans le parler de la population allemande du Banat.

c) Pour faire plaisir à l'enquêteur, les locuteurs s'efforcent d'exprimer, à tout prix, dans l'idiome du village, la totalité des énoncés formulés; faute d'expressions ou de 
mots istro-roumains appartenant au fonds autochtone, ils ont recours à des innovations spontanées, à des créations ad hoc, ou bien ils reprennent des faits de langue plus anciens, appartenant au fonds archaïque, oubliés aujourd'hui par la plupart des sujets parlants: nevręme "mauvais temps", în dim şi-n ne-n dim "dans la fumée ou non"; dupăpódne "après-midi"; prestemấre "après demain", tout comme des numéraux composés exclusivement d'éléments latins doinzéče şi ur, şasezéč şi şåpte, employés à la place des formes usuelles dvåiiset şi ur, şestdéset şi şåpte. Au lieu de cr. vişe, certaines gens plus âgés ont de nouveau recours à la formule, moins employée, mai mund. Il est à remarquer que de telles innovations lexicales, de tels syntagmes réactualisés apparaissent dans les textes enregistrés après quelques instants d'hésitation, ou bien ils sont accompagnés d'explications supplémentaires en croate ou en istro-roumain.

d) Dans toute une série de syntagmes on peut souvent constater un phénomène d'induction réciproque entre les constituants syntagmatiques. Par exemple, dans les syntagmes čúda ómir et vişe l'údi, les premiers termes (čúda-terme croate appartenant au fonds istro-roumain plus ancien et, respectivement, vişe - acquisition récente provenant du croate) manifestent des préférences combinatoires diférentes par rapport aux termes seconds, le premier (čúda) sélectant ómir-mot appartenant au fonds roumain ancien, et vişe le croate l'údi. Cf. l'expression Ne sámo îo, négo vişe l'údi, où le syntagme croate vişe l'údi est amené par le contexte syntaxique spécifiquement croate constitué par les corrélatifs ne sámo - négo.

Un exemple éloquent pour la fréquence de ce phénomène dans les réponses des locuteurs aux différentes questions est constitué par le passage-dans une même phrase du syntagme stára ţârcva au syntagme mixte stára beséreca et ensuite à la forme betấra beséreca, intégralement constituée d'éléments roumains. Ce phénomène apparaît également comme une réaction du sujet parlant à la perplexité qu'on peut lire sur le visage de l'enquêteur lors de l'énonciation du premier syntagme:

"Că ş-íča-n vârh de sélişte sus av ţấrcva, stára besęreca, $(\varepsilon)$ betắra beséreca ... e şi d-atúnče pac a făcút. Nu ştívu dúpă câtă vręeme av jos verít, oånč av čåoåta bisęreca făcút, če-i ân Múne" (Mate Sankovit", 78 ans, Jeiăn).

On peut y remarquer la concurrence des constructions parallèles du croate et de l'istro-roumain, le passage graduel du syntagme croate au syntagme istro-roumain, réalisé tout d'abord par la substitution du nom (tấrcva - biséreca), et ensuite de l'adjectif (stára - betăra). Cet exemple illustre en même temps la facilité avec laquelle les Istro-Roumains-les vieux tout comme les plus jeunes-, réalisent le passage d'un cadre à l'autre. Voici un autre exemple similaire:

"... Åm trei fečór: doi múşki şi o jénska, doi fil' şi-o fíl'e doi âs ânsuråţ s-av ânsuråt. Ur åre fil', ur fil'. E ấncă-i fil'’a de meritåt". (Doričit" Anton, 55 ans, Jeiăn).

e) Par ses différents paramètres, l'acte de communication impose au sujet parlant une certaine conduite et un certain comportement verbal. Le rapport entre le lexique autochtone et le lexique emprunté change-dans des textes variables comme 
dimensions-en fonction du thème abordé (du domaine des préoccupations familiales, des soucis quotidiens du ménage, ou bien du domaine social-administratif, politique, culturel, juridique, etc.), en fonction également de la situation et du moment de la journée (à la table, pendant le travail ou pendant une pause, l'après - midi ou le soir, au café ou devant un verre de vin, au moment des présentations ou en visite), du cadre où la discussion a lieu (entre deux ou plusieurs locuteurs, au restaurant, dans la rue ou à la maison, en famille), en fonction finalement de l'état d'âme des locuteurs (bonne ou mauvaise humeur, joie ou colère).

Dans de telles situations on passe insensiblement du code istro-roumain au code croate et vice-versa, dans des unités de texte plus réduites (mots, syntagmes) ou plus étendues (propositions, phrases). Certes, de tels passages d'un registre linguistique à l'autre sont dus aussi à l'attitude des sujets parlant un certain idiome (restreint comme sphère d'emploi) à l'égard d'un étranger quelconque, dans leur désir d'être mieux compris; ils peuvent s'expliquer aussi par l'habitude des locuteurs à employer, dans la conversation avec un étranger, une autre langue que le parler local.

Cf. quelques exemples:

"ie ţéla sédmiţa ( $(\varepsilon$ čå-i tótile t"édăn ("c'est la semaine tout entière"); bílo famíliịe bílo drújstvo, bílo če ("soit la famille, soit la société, quoi que ce soit"); gótovo ine (il est prêt); préco dévet let ("dans neuf ans"); za vręme ráta ("pendant la guerre"); doublé dans le même contexte par l'expression istro-roumaine când a fost ósta; ni i ie nepocrétna, négo bólna ináco ("elle n'est pas clouée au lit, mais elle est très malade"); dóşao posiéti (doublé dans la même phrase par l'istro-roumain verit-a posiéti); ie ópet hârvátski (c'est encore du croate - la réplique est souvent employée lorsque le sujet parlant constate tout de suite que dans le texte parlé s'est glissé un mot croate remplaçant l'élément istro-roumain oublié au moment de l'énonciation). Cf. également: ma rázlica ịe "il y a une différence"; ie ópasno "c'est dangereux"; ştéta čúda "grands dommages".

f) On devrait également mentionner les expressions-clichés étroitement liées aux autres situations et ayant les mêmes justifications; ces expressions appartiennent au croate mais, dans diverses circonstances, elles apparaissent dans le texte istro-roumain comme des séquences hétéroglottes: Aĭ, l'údi, Isúse!; Siromácu!; A, deţo!; táco néşto; víse nícad; íáco bólna i ie; za vréme ráta; bog zna et d'autres.

On peut observer que, dans la plupart des cas, les informateurs s'expriment avec facilité, d'une manière cohérente, en employant un grand nombre de constructions lexico-syntaxiques appartenant au fonds istro-roumain ancien, lorsque la relation porte sur leur famille et leurs occupations quotidiennes (voir les exemples ci-dessus) ou bien lorsqu'ils reproduisent des textes plus anciens, consacrés par le temps, poésies, chansons, proverbes et dictons.

Par exemple: Més-åm oånče şi colę / Pre zeléne iåråbe,/

Ke voi vę́de feétiţa / Cum e्na blåya végl'a./ 
Féto, feéto, feétiţe, / Namajéţ to úşiţa,/

Ke-ț va verí fråieru, / Rójițe la droåculuị!!/

Cf. roum.: "Mers-am pe-aici şi colea / Prin iarba verde/

Ca să văd fetiţa / Cum păzeşte vitele/

Fato, fato, fetiţo, / Unge-ţi uşiţa!/

Că-ţi va veni drăguţul / Florile dă-le dracului!/"

(Turkovit" Draga, 57 ans, Jeiăn).

Cf. également: Se nu-i måčca, şóreči jócu ân cåsă "Le chat parti, les souris dansent"; Ómu nícad nu-i bétăr "L’homme n'est jamais trop vieux"; Mai bíre uåstaz. óvu négo mấre galíra "Un tiens vaut mieux que deux tu l'auras"; Muşåta fę́te, e grúmbo se pórta "Elle est belle fille, mais elle a de mauvais comportements". (Dora Tvečić, 60 ans, Suşnieviţa).

Cependant, lorsque les locuteurs sont sollicités dans un dialogue construit ad-hoc, portant sur des thèmes sociaux ou administratifs, politiques ou économiques, il leur arrive de passer, insensiblement, du registre istro-roumain au registre croate, auquel ils sont tout aussi habitués, si bien qu'il est difficile d'établir chaque fois, dès le début, si ces passages sont accidentels ou bien si l'on a affaire à des éléments et à des structures du système istro-roumain actuel, issus du mélange des deux langues. Dans le cas où les éléments croates insérés dans un texte quelconque ont une certaine étendue, nous pensons qu'il ne s'agit pas d'un mélange de langues, mais plutôt que, dans certaines situations, les sujets parlants abandonnent de manière consciente (voir les situations a,b,d,e décrites ci-dessus) ou inconsciente (voir les situations $\mathrm{c}$ et d) leur idiome au profit de la langue officielle, pour des raisons différentes (l'intention des locuteurs d'être mieux compris par l'enquêteur, la disposition des sujets dans certaines situations, etc.), tout à fait justifiées du point de vue psycholinguistique.

On peut donc observer que la pression du croate sur le système lexical istro-roumain se manifeste plus ou moins intensément en fonction du domaine thématique et du contexte situationnel plus large (a) soit comme transfert de structures lexicales et syntaxiques intégrales, composées d'éléments sous leur forme d'origine, sans aucune adaptation au système istro-roumain, (b) soit comme transfert de structures et d'éléments partiels. Dans ce dernier cas celui des combinaisons lexicales "mixtes", la résistence de l'idiome à la pression alloglotte est évidente, tout comme sa tendance à conserver son identité par une "opposition active" aux dislocations massives et le maintien de ses particularités spécifiques jusqu'à la disparition des derniers sujets parlant cet idiome. Ces combinaisons mixtes sont éloquentes pour la manière dont l'idiome subit et exprime, le long des siècles, la pression alloglotte, dans notre cas l'influence de trois langues étrangères. 


\section{OPOMBE K SEDANJEMU ISTROROMUNSKEMU BESEDIŠČU}

Analiza precejšnjega števila besedil, ki jih je avtor v letu 1982 nabral v Žejanah in v Sušnjevici (Hrvaška Istra) in pozneje objavil v delu Texte istroromâne şi glosar (Tipografia Universitâţii din Timişoara, 1987) potrjuje prepričanje, da je vpliv hrvaškega jezika, $v$ njegovi čakavski različici, $v$ istroromunščini zaznaven $v$ vseh delih tega govora, celo v morfosintaksi. Pri tem ugotavljamo, da teme vsakdana obvladuje staro romansko besedišče. Besedila s socialno-administrativno ali pa politično-ekonomsko tematiko pa imajo veliko slovanskih izrazov, hrvaških ali slovenskih.

Prispevek želi natančneje predstaviti situacije, $v$ katerih sta si slovanski in romanski leksikalni in sintaktični sistem še posebej $v$ nasprotju, obenem pa želi opozoriti na jezikovne in zunajjezikovne dejavnike, na sociolingvistične in psiholingvistične momente, kolikor ti narekujejo izbiro slovanskih, torej hrvaških ali slovenskih jezikovnih prvin.

Avtor izhaja iz prepričanja, da so pripadniki istroromunskega življa v celoti dvojezični, da pa je moč ugotoviti jezikovne hrvatizme tudi v sproščenem govoru. Želi pa razlikovati med resničnimi izposojenkami iz hrvaščine in tistimi, kjer gre za prehod iz enega jezikovnega koda $v$ drugi. Vsekakor pa je $v$ istroromunskem osnovnem besedišču mogoče najti visoko število besed latinskega izvora. 